\title{
TempoExpress, a CBR Approach to Musical Tempo Transformations
}

\author{
Maarten Grachten, Josep Lluís Arcos, and Ramon López de Mántaras \\ IIIA, Artificial Intelligence Research Institute, \\ CSIC, Spanish Council for Scientific Research, \\ Campus UAB, 08193 Bellaterra, Catalonia, Spain, \\ \{maarten, arcos, mantaras\}@iiia.csic.es, \\ http://www.iiia.csic.es \\ Application Paper \\ Keywords: applications of CBR, generative reuse
}

\begin{abstract}
In this paper, we describe a CBR system for applying musically acceptable tempo transformations to monophonic audio recordings of musical performances. Within the tempo transformation process, the expressivity of the performance is adjusted in such a way that the result sounds natural for the new tempo. A case base of previously performed melodies is used to infer the appropriate expressivity. Tempo transformation is one of the audio post-processing tasks manually done in audiolabs. Automatizing this process may, therefore, be of industrial interest.
\end{abstract}

\section{Introduction}

In this paper we describe a CBR system, TempoExpress, that automatically performs musically acceptable tempo transformations. This paper significantly extends previous work [1], that addressed the process of performance annotation, a basic step to construct the cases needed in the CBR system described now.

The problem of changing the tempo of a musical performance is not as trivial as it may seem. When a musician performs a musical piece at different tempos, the performances are not just time-scaled versions of each other, as if the same performance were played back at different speeds. Together with the changes of tempo, variations in musical expression are made [3]. Such variations do not only affect the timing of the notes, but can also involve for example the addition or deletion of ornamentations, or the consolidation/fragmentation of notes. Apart from the tempo, other domain specific factors seem to play an important role in the way a melody is performed, such as meter, and phrase structure.

Tempo transformation is one of the audio post-processing tasks manually done in audio-labs. Automatizing this process may, therefore, be of industrial interest.

In section 2, we will present the overall structure of TempoExpress. In section 3, we briefly explain the processes involved in case and problem representation. Section 4 describes the crucial problem solving phases of the CBR 


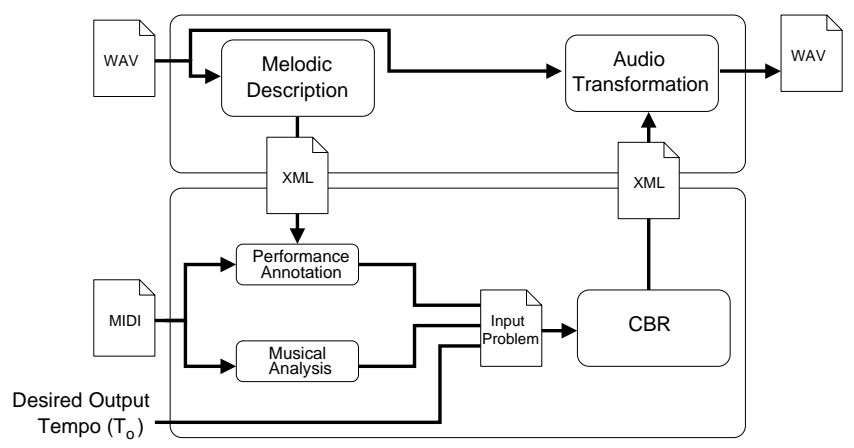

Fig. 1. Overview of the basic TempoExpress components

mechanism, retrieval and reuse. In section 5, some initial results are presented. Conclusions and future work are presented in section 6 .

\section{Overview of TempoExpress}

TempoExpress consists of three main parts and two additional parts. The main parts are the melodic description module, the CBR problem solving module, and the audio transformation module. The additional parts are the performance annotation module and the musical analysis module (see figure 1). The melodic description module generates a melodic description of the input recording, that represents information about the performance on a musical level. This information is used together with the score of the performed melody (as a MIDI file), and the desired tempo of the output performance, to construct an input problem. CBR is then applied to obtain a solution for the problem in the form of a melodic description of the new performance. The audio transformation produces an audio file, based on the original audio and the new melodic description.

Since the main information in the input problem and the cases (the melodic material of the score and the annotated performance) is of sequential nature, we apply edit distance techniques in the retrieval step, as a means to assess similarities between the cases and the input problem. In the reuse step we employ constructive adaptation [12], a reuse method for synthetic tasks. This method constructs a solution to a problem by searching the space of partial solutions for a complete solution that satisfies the solution requirements of the problem.

\subsection{Melodic Description and Audio Transformation}

The melodic description and audio transformation are not part of the research reported here. These processes are being implemented within a common research project by members of the Music Technology Group (MTG) of the Pompeu Fabra University, using signal spectral modeling techniques (see [13,4] for a detailed description). The output of the melodic description process (and input of the 
audio transformation process), is a description of the audio in $X M L$ format, that adheres to (and extends) the MPEG7 standard for multimedia description.

\section{Case/Problem Representation}

In this section, we will explain the various aspects of the construction of cases from available information. To construct a case, a score (in MIDI format) is needed. This score is represented internally as a sequence of note objects, with the basic attributes like pitch, duration and temporal position. This score is analyzed automatically to obtain a more abstract representation of the melody, called I/R representation. This procedure is explained in subsection 3.1. Furthermore, an input performance at a particular tempo is needed. The performance is not stored literally, but rather a performance annotation is constructed to describe how the elements from the performance relate to the elements from the score. This procedure is explained in detail in [1], and is briefly reminded in subsection 3.2. The performance annotation is stored as a solution, associated to a particular input description that applies to the performance (in our case, the tempo of the performance). Lastly, the desired output tempo is also included as a part of the problem description, specifying what the solution should be like.

\subsection{Music Analysis}

To prepare cases, as well as the input problem, music analysis is performed on the musical score that was provided. The analysis is used in the problem solving process, for example to segment musical phrases into smaller groups of notes, and to perform retrieval of cases. The musical analysis is based on a model for melodic structure, that is explained below.

The Implication/Realization Model Narmour [10,11] has proposed a theory of perception and cognition of melodies, the Implication/Realization model, or I/R model. According to this theory, the perception of a melody continuously causes listeners to generate expectations of how the melody will continue. The sources of those expectations are two-fold: both innate and learned. The innate sources are 'hard-wired' into our brain and peripheral nervous system, according to Narmour, whereas learned factors are due to exposure to music as a cultural phenomenon, and familiarity with musical styles and pieces in particular. The innate expectation mechanism is closely related to the gestalt theory for visual perception [7,8] and for audition [2]. Gestalt theory states that perceptual elements are (in the process of perception) grouped together to form a single perceived whole (a 'gestalt'). This grouping follows certain principles (gestalt principles). The most important principles are proximity (two elements are perceived as a whole when they are perceptually close), similarity (two elements are perceived as a whole when they have similar perceptual features, e.g. color or form, in visual perception), and good continuation (two elements are perceived as a whole if one is a 'good' or 'natural' continuation of the other). Narmour 


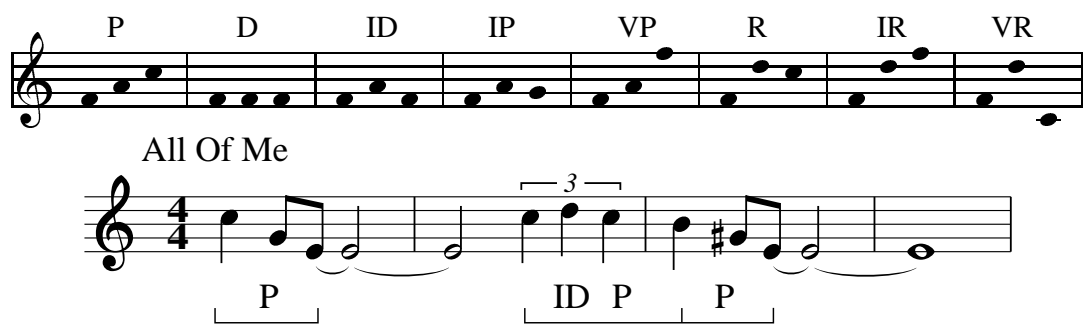

Fig. 2. Top: Eight of the basic structures of the I/R model. Bottom: First measures of All of Me, annotated with I/R structures

claims that similar principles hold for the perception of melodic sequences. In his theory, these principles take the form of implications: Any two consecutively perceived notes constitute a melodic interval, and if this interval is not conceived as complete, or closed, it is an implicative interval, an interval that implies a subsequent interval with certain characteristics. In other words, some notes are more likely to follow the two heard notes than others. Two main principles concern registral direction and intervallic difference. The principle of registral direction states that small intervals imply an interval in the same registral direction (a small upward interval implies another upward interval, and analogous for downward intervals), and large intervals imply a change in registral direction (a large upward interval implies another upward interval and analogous for downward intervals). The principle of intervallic difference states that a small (five semitones or less) interval implies a similarly-sized interval (plus or minus 2 semitones), and a large intervals (seven semitones or more) implies a smaller interval.

Based on these two principles, melodic patterns can be identified that either satisfy or violate the implication as predicted by the principles. Such patterns are called structures and labeled to denote characteristics in terms of registral direction and intervallic difference. Eight such structures are shown in figure 2(top). For example, the $\mathrm{P}$ structure ('Process') is a small interval followed by another small interval (of similar size), thus satisfying both the registral direction principle and the intervallic difference principle. Similarly the IP ('Intervallic Process') structure satisfies intervallic difference, but violates registral direction.

Additional principles are assumed to hold, one of which concerns closure, which states that the implication of an interval is inhibited when a melody changes in direction, or when a small interval is followed by a large interval. Other factors also determine closure, like metrical position (strong metrical positions contribute to closure, rhythm (notes with a long duration contribute to closure), and harmony (resolution of dissonance into consonance contributes to closure). The closure in each of these dimensions add up to the total closure. The occurrence (and degree) of closure at a given point in the melody determines where the structures start and end. For example, on a note where strong closure appears (e.g. closure in meter, harmony and rhythm at the same time), the interval between that note and the next will not be perceived as implicative, and 
therefore there is no structure describing that interval. When no closure occurs at all, every interval implies a new interval, and since the structures describe two subsequent intervals, this causes a chaining, or overlapping of structures.

We have designed an algorithm to automate the annotation of melodies with their corresponding I/R analyses. The algorithm implements most of the 'innate' processes mentioned before. The learned processes, being less well-defined by the I/R model, are currently not included. Nevertheless, we believe that the resulting analysis have a reasonable degree of validity. An example analysis is shown in figure 2(bottom). This example shows various degrees of structure chaining: the first two structures (P and ID) are not chained, due to strong closure (meter and rhythm); the second pair of structures (ID and P) are strongly chained (sharing two notes, one interval), because closure is inhibited by 'ongoing' rhythms (like triplets); the last pair of structures ( $\mathrm{P}$ and $\mathrm{P}$ ) are chained by one note, because of weak closure (only in meter).

\subsection{Performance Annotation}

In addition to the score and its musical analysis, the cases in the case base, as well as the problem specification, contain a performance of that score by a musician. The raw format of the performance is an audio file. Using the melodic description mechanism described in section 2.1, we obtain a description (conforming to the MPEG-7 standard) of the performance, in XML format. This description contains a sequence of note descriptors, that describe the features like start and end times, pitch, energy of the notes, as they were detected in the audio file. In order to be informative, the sequence of note descriptors is to be mapped to the notes in the score, since this mapping expresses how the score was performed. For example, it allows us to say that a particular note was lengthened or shortened, or played early or late.

But the mapping between score notes and performed notes does not necessarily consist of just 1-to-1 mappings. Especially in jazz performances, which is the area on which we will focus, performers often favor a 'liberal' interpretation of the score. This does not only involve changes in expressive features (like lengthening/shortening durations) of the score elements as they are performed, but also omitting or adding notes. Thus, one can normally not assume that the performance contains a corresponding element for every note of the score, neither that every element in the performance corresponds to a note of the score. Taking these performance liberties into account, a description of a musical performance could take the form of a sequence of add/delete/transform operations that are applied to the score elements in order to obtain the performance.

From this perspective the edit distance [9] is very useful, since it allows us to obtain an optimal alignment from score to performance, given a set of operations. The set of allowed edit operations we use is a slight revision of the set proposed by Arcos et al. [1]. It includes:

Transformation Representing the reproduction of a score note, possibly with several kinds of transformations, such as change of pitch, duration and temporal position 
Insertion Representing the occurrence of a performance note that does not correspond to any score note

Ornamentation A special case of insertion, where the inserted note functions as a grace note (a very short note played as a lead-in to the next note)

Deletion Representing the occurrence of a score note that does not correspond to any performance note

Fragmentation Representing the reproduction of a score note by playing two or more shorter notes (adding up to the same total duration)

Consolidation Representing the reproduction of two or more score notes by playing a single longer note (whose duration equals the sum of the score note durations)

We defined the costs of these operations as functions of the note attributes (pitch, duration and onset). However, rather than fixing the relative importance of the attributes (as in [1]), we parametrized the cost functions to be able to control the importance of each of the note attributes in each of the cost functions, and the relative costs of edit operations. This setup enables us to tune the performance alignment algorithm to produce alignments that correspond to intuitive human judgment. We have used a genetic algorithm [6] to tune the parameters of the cost functions. The parameter settings were evaluated by comparing the alignments they produced for a set of performances, to manually constructed alignments.

\section{Problem Solving}

In this section, we will explain the steps taken to transform the performance presented as input into a performance of the same score at a different tempo. The first step is the retrieval of relevant cases from the case base. In the second step, the retrieved cases are selectively used to obtain a new sequence of performance events. This sequence can then be used to modify the XML description of the performance. Based on this modified description, the original audio file is transformed to obtain the final audio of the performance at the desired tempo.

\subsection{Retrieval}

The goal of the retrieval step is to form a pool of relevant cases, that can possibly be used in the reuse step. This done in the following three steps: firstly, cases that don't have performances at both the input tempo and output tempo are filtered out; secondly, those cases are retrieved from the case base that have phrases that are I/R-similar to the input phrase; lastly, the retrieved phrases are segmented. The three steps are described below.

Case filtering by tempo In the first step, the case base is searched for cases that have performances both at the tempo the input performance was played, and the tempo that was specified in the problem description as the desired 
output tempo. The matching of tempos need not be exact, since we assume that there are no drastic changes in performance due to tempo within small tempo ranges. For example, a performance played at 127 beats per minute (bpm) may serve as an example case if we want to construct a performance at 125 bpm. We currently allow an (arbitrarily chosen) tempo deviation of $5 \mathrm{bpm}$ in both directions. Obviously, increasing the tolerance for deviation of tempo will decrease the validity of the solution obtained.

I/R based retrieval In the second step, the cases selected in step 1 are assessed for melodic similarity to the score specified in the problem description. In this step, the primary goal is to rule out the cases that belong to different styles of music. For example, if the score in the problem description is a ballad, we want to avoid using a bebop theme as an example case. Note that the classification of musical style based on just melodic information (or derived representations) is far from being an established issue. Nevertheless, there is some initial evidence [5] that the comparison of melodic material at different levels of abstraction yields different degrees of discriminatory power. For example comparing on the most concrete level (comparing individual notes) is a good way to find out which melodies in a set are nearly identical to a particular target melody. But if the set of melodies does not contain a melody nearly identical to the target, the similarity values using this measure are not very informative, since they are highly concentrated in a single value. On the other hand, comparisons based on more abstract descriptions of the melody (e.g. melodic contour, or $\mathrm{I} / \mathrm{R}$ analyses), tend to produce a distribution of similarity values that is spread out through the spectrum more equally. Thus, these measures tell us in a more informative way how similar two melodies are (with respect to the other melodies in the set), even if they are considerably different. As a consequence, a melodic similarity measure based on an abstract representation of the melody seems a more promising approach to separate different musical styles.

We use the I/R analysis of the melodies to assess similarities. The measure used is an edit-distance, with edit-operations (and their corresponding costs) that operate on sequences of $\mathrm{I} / \mathrm{R}$ structures. The case base is ranked according to similarity with the target melody, and the subset of cases with similarity values above a certain threshold are selected. The resulting set of cases will contain phrases that are roughly similar to the input score.

Segmentation In this step, the melodies that were retrieved in the second step are segmented. The segmentation is based on the $\mathrm{I} / \mathrm{R}$ representation of the melodies. The melodies are split at every point where the overlap of two $\mathrm{I} / \mathrm{R}$ structures is less than two notes (see section 3.1). The resulting segments usually correspond to the musical motifs that constitute the musical phrase, and are used as the units for the stepwise construction of the output performance.

As an example, figure 3 displays the segmentation of the first phrase of 'All of Me' (the complete phrase is shown in figure 2). 

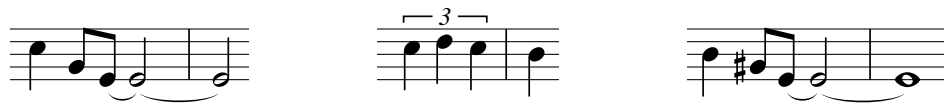

Fig. 3. Segmentation of the first phrase of 'All of Me', according to I/R structures. The segments correspond to single I/R structures, or sequences of structures if they are strongly chained (see subsection 3.1 )

\subsection{Reuse}

In the reuse step a performance of the input score is constructed at the desired tempo, based on the input performance and the set of retrieved phrase segments. This step is realized using constructive adaptation [12], a technique for reuse that constructs a solution by a best-first search through the space of partial solutions. In this subsection, we will first explain briefly how the reuse step can in general be realized as best-first search, and then we will explain how we implemented the functions necessary to make the search-algorithm operational in the context of performance transformation.

In constructive adaptation, partial solutions of the problem are represented as states. Furthermore, a function HG must be defined for generating a set of

successor states for a given state. The state space that emerges from this function and the state that represents the empty solution (generated by a function Initial-State), is then searched for a complete solution that satisfies certain constraints (through a function Goal-Test). The resulting state is transformed to a real solution by a function SAC. The order of expansion of states is controlled by a function HO that orders the states in a best-first manner. The search process is expressed in pseudo code below.

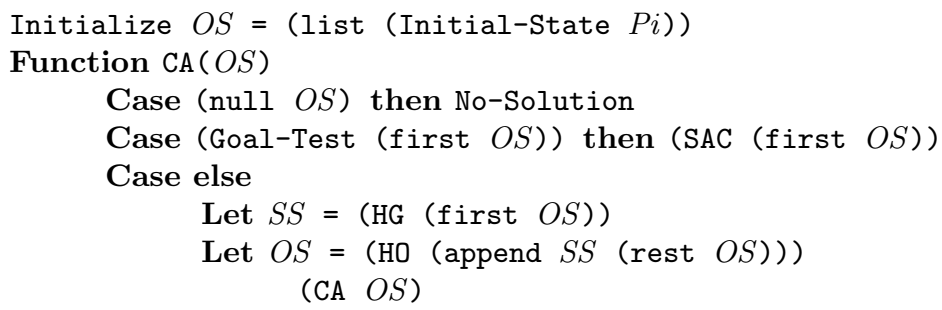

Fig. 4. The search process of constructive adaptation expressed in pseudo code. Functions HG and HO are Hypotheses Generation and Hypotheses Ordering. Variables $O S$ and $S S$ are the lists of Open States and Successor States. The function SAC maps the solution state into the configuration of the solution. The function Initial-State maps the input problem description $P i$ into a state. From Plaza and Arcos [12]

We explain our implementations of the functions Initial-State, HG, HO, Goal-Test, and SAC below. 


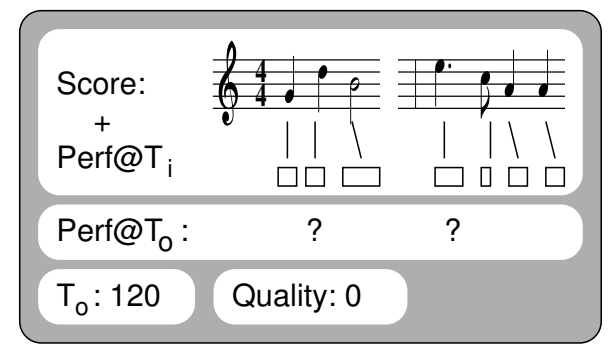

Fig. 5. Example of an initial state in Constructive Adaptation. $T_{i}$ is the tempo of the input performance; $T_{o}$ is the desired output tempo

Initial-State The function Initial-State returns a state that is used as the starting point for the search. It takes the input problem description (the score, analysis, input-performance, and desired output tempo) as an argument. In our case, the state contains a sequence of score segments, and a slot for storing the corresponding performance segments (none of which is filled in the initial state, obviously). Furthermore, there is a slot that stores the quality of the partially constructed performance, as a number. We will explain the derivation of this number in the next subsection. Figure 5 shows the initial state for a short musical fragment (containing two segments).

Hypothesis-Generation (HG) The Hypothesis-Generation function takes a state as an argument and tries to find a sequence performance events for one of the unprocessed score segments in the state. We will illustrate this procedure step by step, using the first segment of the initial state in figure 5 as an example. The steps are presented graphically in figure 7 (at the last page of this paper).

The first step is to find the segment in the pool of retrieved melodic segments that is most similar to the input score segment. The similarity is assessed by calculating the edit-distance between the segments (the edit distance now operates on notes rather than on $\mathrm{I} / \mathrm{R}$ structures, to have a finer grained similarity assessment). A mapping between the input score segment and the best matching retrieved segment is made.

In the second step, the performance annotation events (see subsection 3.2 and [1]) corresponding to the relevant tempos are extracted from the retrieved segment case and the input problem specification (both the input tempo $T_{i}$ and the output tempo $T_{o}$ for the retrieved segment case, and just $T_{i}$ from the input problem specification).

The third step consists in relating the annotation events of the retrieved segment to the notes of the input segment, according to the mapping between the input segment and the retrieved segment, that was constructed in the first step. For the notes in the input segment that were mapped to one or more notes in the retrieved segment, we now obtain the tempo transformation from $T_{i}$ to $T_{o}$ that was realized for the corresponding notes in the retrieved segment. It is 
also possible that some notes of the input segment could not be matched to any notes of the retrieved segment. For such notes, the retrieved segment can not be used to obtain annotation events for the output performance. Currently, these gaps are filled up by directly transforming the annotation events of the input performance (at tempo $T_{i}$ ) to fit the output tempo $T_{o}$ (by scaling the duration of the events to fit the tempo). In the future, more sophisticated heuristics may be used.

In the fourth step, the annotation events for the performance of the input score at tempo $T_{o}$ are generated. This is done in a note by note fashion, using rules that specify which annotation events can be inferred for the output performance of the input score at $T_{o}$, based on annotation events of the input performance, and the annotation events of the retrieved performances (at $T_{i}$ and $T_{o}$ ). To illustrate this, let us explain the inference of the Fragmentation event for the last note of the input score segment (B)in figure 7 . This note was matched to the last two notes $(\mathrm{A}, \mathrm{A})$ of the retrieved segment. These two notes were played at tempo $T_{i}$ as a single long note (denoted by the Consolidation event), and played separately at tempo $T_{o}$. The note of the input segment was also played as a single note at $T_{i}$ (denoted by a Transformation event rather than a Consolidation event, since it corresponds to only one note in the score). To imitate the effect of the tempo transformation of the retrieved segment (one note at tempo $T_{i}$ and two notes at tempo $T_{o}$ ), the note in the input segment is played as two shorter notes at tempo $T_{o}$, which is denoted by a Fragmentation event $(\mathrm{F})$.

In this way, adaptation rules were defined, that describe how the tempo transformation of retrieved elements can be translated to the current case. In figure 7, two such rules are shown. If the antecedent part matches the constellation of annotation events, the tempo transformation in the consequent part can be applied. It can occur that the set of rules contains no applicable rule for a particular constellation, in particular when the performances at $T_{i}$ of the retrieved note and the input note are too different. For example, if the score note is played as a Transformation event, but the retrieved note is deleted in the performance at $T_{i}$, then the performances are too different to make an obvious translation. In this case, the annotation events from the input performance are transformed in the same way as in the case where no corresponding note from the retrieved segment could be found (see the third step of this subsection).

The mismatch between the input segment and the retrieved segment and the inability to find a matching adaptation rule obstructs the use of case knowledge to solve the problem and forces TempoExpress to resort to default mechanisms. This will affect the quality of the solution. To reflect this, the value of the quality slot of the state (see figure 5) is calculated as the number of input score notes for which annotation events could be inferred from retrieved cases, divided by the total number of notes processed so far (that is, the sum of all notes in the processed input segments, including the current input segment).

Hypothesis-Ordering (HO) The Hypothesis-Ordering function takes a list of states (each one with its partial solution) and orders them so that the states 
with the most promising partial solutions come first. For this ordering, the quality value of the states is used. In our current implementation, the quality value is only determined by one factor, roughly the availability of appropriate cases. Another factor that should ideally influence the quality of the states is the "coherence' of the solution. For example, if the notes at the end of one segment were anticipated in time (as a possible effect of a Transformation event), then anticipation of the first notes of the next segment will not have the typical effect of surprise, since the listener will experience the performance as being shifted forward in time, instead of hearing a note earlier than expected. We are currently incorporating the detection and evaluation of such phenomena into the Hypothesis-Ordering function, so that this functionality will soon be available.

Goal-Test The Goal-Test function is called on the best state of an ordered list of states to test if the solution of that state is complete and satisfies the constraints imposed upon the desired solution. The completeness of the solution is tested by checking if all segments of the input score have a corresponding segment in the performance annotation for the output tempo. The constraints on the solution are imposed by requiring a minimal quality value of the state. In our case, where the quality value represents the ratio of notes for which annotation events were obtained using retrieved cases (a value between 0 and 1), the quality value is required to be superior or equal to 0.8 .

State-to-Solution (SAC) The State-to-Solution function takes the state that passed the goal-test and returns a solution to the input problem. This step consists in building a complete performance annotation from the annotation events for the score segments (basically concatenation of the events). The new performance annotation is used to adapt the XML description of the original audio file, by changing attribute values, and possibly deleting and inserting new note descriptors. Finally, the audio transformation module (which is under development) generates a new audio file, based on the new XML description.

\subsection{Retain}

When the solution that was generated is satisfying to the listener, and when the quality of the solution is high (that is, default adaptation operations have been scarcely used, or not at all), it is retained as a case that includes the input score, the input performance, and the newly generated performance.

\section{$5 \quad$ Results}

Although the TempoExpress is operational, there are some components that need improvement. In particular, the case base is still of limited size (it contains seven different phrases from two different songs, played at approximately ten different tempos). As a result, the range of melodies for which TempoExpress can 


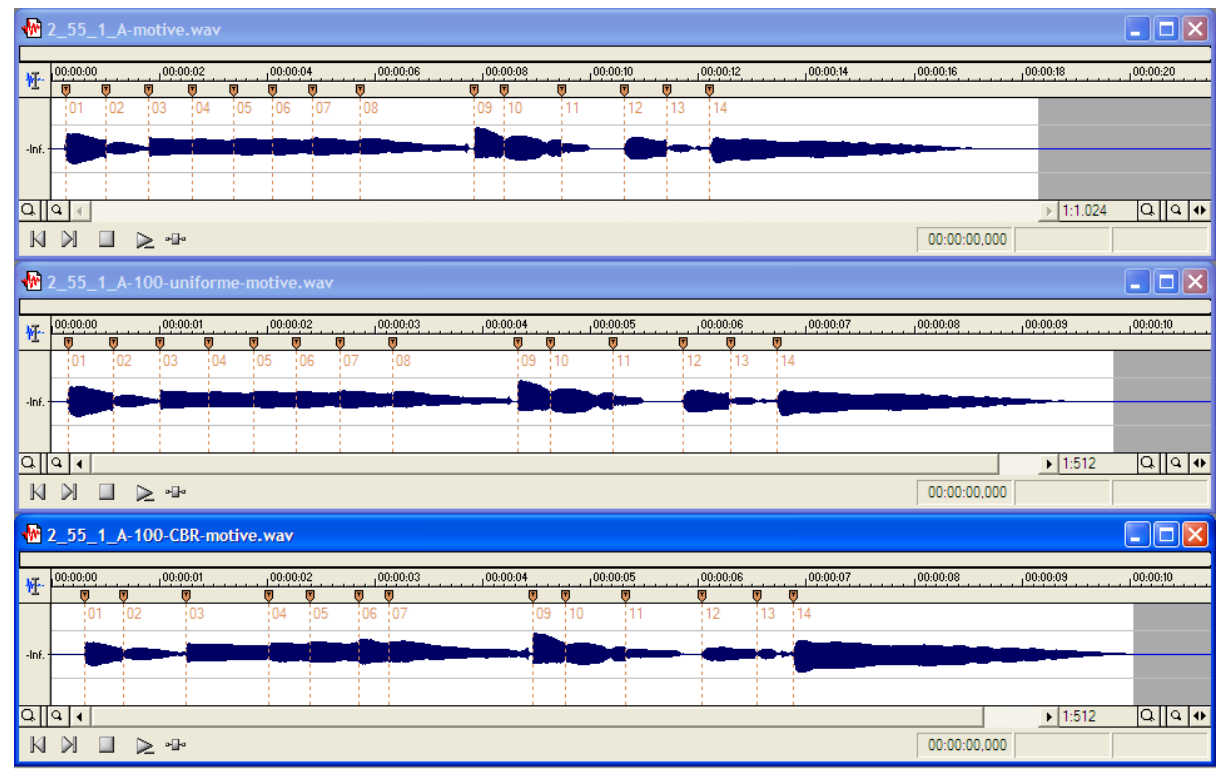

Fig. 6. Audio signals of a part of the first phrase of Once I Loved. The upper view shows original sound file ( $55 \mathrm{bpm})$, the middle view shows a tempo transformation by uniform time stretching, and the lower view shows a tempo transformation using the CBR system. The vertical lines indicate the positions of the note onsets

generate a good tempo transformation is very small, since for many melodies, the case base does not contain a sufficiently similar melody. Nevertheless, we have generated some initial results, by leaving one phrase (and its performances) out of the case base and performing a tempo transformation for that phrase using the other cases in the case base. The original performance of the phrase was at a tempo of 55 beats per minute (bpm), and using the CBR system, the performance was transformed to a tempo of $100 \mathrm{bpm}$. For comparison, the tempo transformation was also realized using uniform time stretching of the original sound file (i.e. the durations of all notes in the original performance are lengthened by a single scaling factor, while leaving the pitches of the notes unchanged). Figure 6 shows the audio signals of the original sound, and the two transformations. Notable differences between the two transformations occur in the notes 3 to 9 (the numbered vertical lines in the views indicate the start of the notes). Notice that in the CBR transformation, the eighth note is missing, due to a consolidation. Furthermore, those notes have considerable variations of duration in the CBR transformation, whereas they are more regularly played in the uniform transformation (as in the original). Slight changes in the dynamics can also be observed, e.g in note 1 and 12. The sound files from the example are publicly available in mp3-format, through the world-wide web ${ }^{1}$.

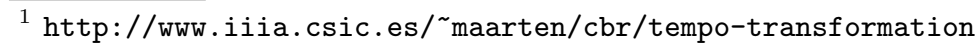




\section{Conclusions and Future Work}

In this paper, we have described TempoExpress, an application for applying musically acceptable tempo transformations to monophonic audio recordings of musical performances. TempoExpress has a rich description of the musical expressivity of the performances, that includes not only timing deviations of performed score notes, but also represents more rigorous kinds of expressivity such as note ornamentation, consolidation, and fragmentation. Within the tempo transformation process, the expressivity of the performance is adjusted in such a way that the result sounds natural for the new tempo. A case base of previously performed melodies is used to infer the appropriate expressivity.

Future work includes elaborating the reuse step, to put more musical constraints on the way in which partial solutions can be combined. Also, we intend to add more cases to the case base, to broaden the range of problems that can be satisfyingly solved by the system.

\subsection{Related Work}

To our knowledge, there is no existing application for automatically applying tempo transformation taking into account the musical expression present in the performance. In the field of performance generation using CBR, there is some previous work. Serra et al. [14] have designed SaxEx, a system for adding expressiveness to inexpressive performances of melodies. Some design choices in TempoExpress were adapted from this application. Furthermore, Suzuki has recently presented Kagurame, a CBR system for the expressive performance of a musical score [15]. Although the task of Kagurame is performance generation, rather than performance transformation, some of the subtasks are common, such as performance to score matching, and segmentation of the score. The way in which these tasks are carried out differs however.

\section{Acknowledgments}

This research has been partially supported by the Spanish Ministry of Science and Technology under the project TIC 2003-07776-C2-02 "CBR-ProMusic: Content-based Music Processing using CBR" and EU-FEDER funds. The au-

thors acknowledge the Music Technology Group of the Pompeu Fabra University for providing the melodic description and audio transformation modules.

\section{References}

1. J. Ll. Arcos, M. Grachten, and R. López de Mántaras. Extracting performer's behaviors to annotate cases in a CBR system for musical tempo transformations. In Proceedings of the Fifth International Conference on Case-Based Reasoning (ICCBR-03), 2003.

2. A. S. Bregman. Auditory Scene Analysis. MIT Press, Cambridge, MA, 1990. 
3. P. Desain and H. Honing. Tempo curves considered harmful. In "Time in contemporary musical thought" J. D. Kramer (ed.), Contemporary Music Review. 7(2), 1993.

4. E. Gómez, A. Klapuri, and B. Meudic. Melody description and extraction in the context of music content processing. Journal of New Music Research, 32(1), 2003.

5. M. Grachten, J. Ll. Arcos, and R. López de Mántaras. A comparison of different approaches to melodic similarity, 2002. Second International Conference on Music and Artificial Intelligence (ICMAI).

6. M. Grachten, J. Ll. Arcos, and R. López de Mántaras. Evolutionary optimization of music performance annotation, 2004. submitted.

7. K. Koffka. Principles of Gestalt Psychology. Routledge \& Kegan Paul, London, 1935.

8. W. Köhler. Gestalt psychology: An introduction to new concepts of modern psychology. Liveright, New York, 1947.

9. V. I. Levenshtein. Binary codes capable of correcting deletions, insertions and reversals. Soviet Physics Doklady, 10:707-710, 1966.

10. E. Narmour. The Analysis and cognition of basic melodic structures : the implication-realization model. University of Chicago Press, 1990.

11. E. Narmour. The Analysis and cognition of melodic complexity: the implicationrealization model. University of Chicago Press, 1992.

12. E. Plaza and J. Ll. Arcos. Constructive adaptation. In Susan Craw and Alun Preece, editors, Advances in Case-Based Reasoning, number 2416 in Lecture Notes in Artificial Intelligence, pages 306-320. Springer-Verlag, 2002.

13. X. Serra, J. Bonada, P. Herrera, and R. Loureiro. Integrating complementary spectral methods in the design of a musical synthesizer. In Proceedings of the ICMC'9'7, pages 152-159. San Francisco: International Computer Music Asociation., 1997.

14. X. Serra, R. Lopez de Mantaras, and J. Ll. Arcos. Saxex : a case-based reasoning system for generating expressive musical performances. In Proceedings of the International Computer Music Conference 1997, pages 329-336, 1997.

15. T. Suzuki. The second phase development of case based performance rendering system "Kagurame". In Working Notes of the IJCAI-03 Rencon Workshop, pages 23-31, 2003 


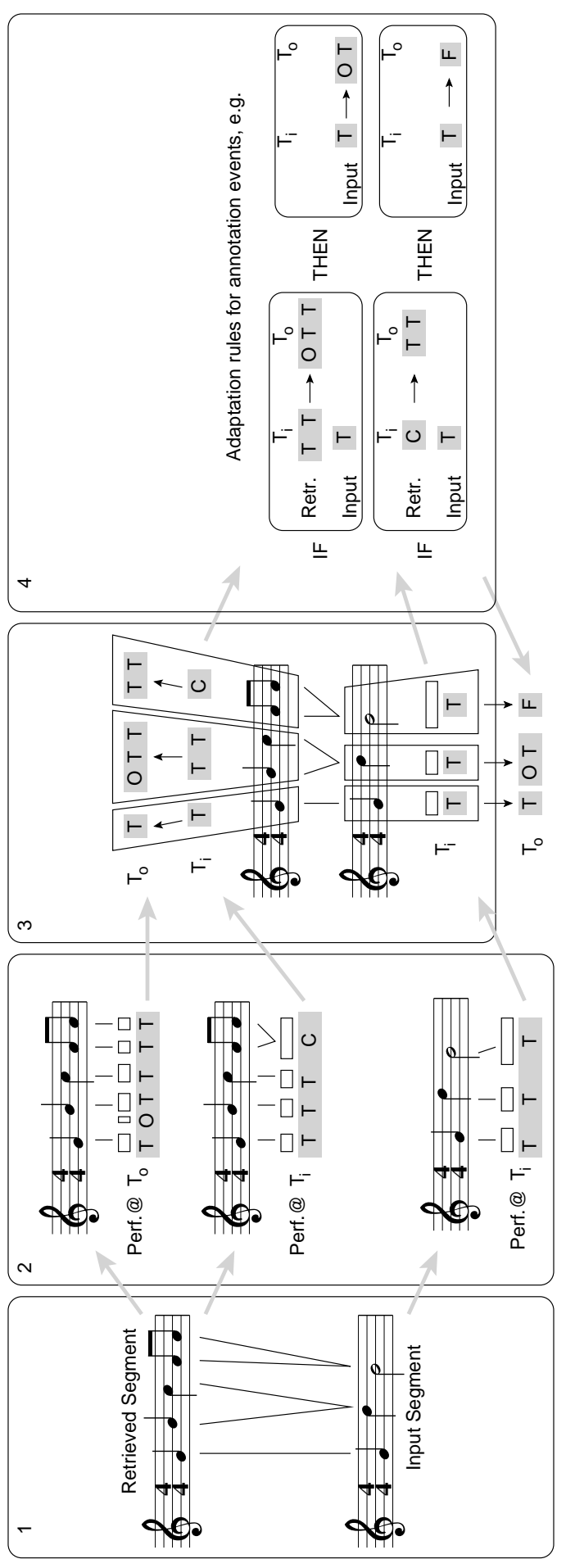

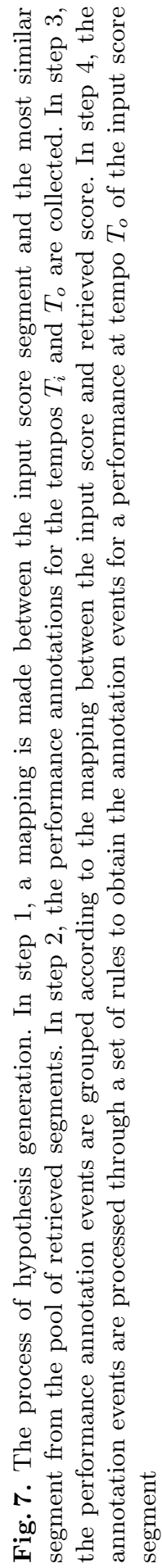

